showed a range of visits and complexity of care. RGHC staff provided $19 \%$ of the care at a cost of $£ 3295$; district nurses $13 \%$, cost $£ 2005$; and formal carers $55 \%$ at a cost of $£ 1344$. 23 GPs visits comprised $4 \%$ of all visits but $15 \%$ of total costs. Macmillan/Marie Curie nurses accounted for just 2\% of visits but $19 \%$ of cost as they stayed overnight. The entire cost of 177 days of care for 17 patients at end of life was $£ 11,814$; i.e. $£ 66.7$ per day as care was not needed every day of each diary period. The average length of the diaries was 10.4 days.

Conclusions The cost of home care seems acceptable, compared to the national average cost of a day in an inpatient specialist palliative care bed at $£ 397-£ 400$ (Data.Gov.UK 2015).

\section{P-154 EMERGENCY ADMISSIONS TO A HOSPICE: CAN HOSPICE AT HOME SERVICES REDUCE THESE?}

Kirsty Lewis, Aparajita Das. Countess Mountbatten Hospice, Southampton, UK

\subsection{6/bmjspcare-2018-hospiceabs.179}

Background Hospice at Home $(\mathrm{H} @ \mathrm{H})$ services aim to provide at-home care whilst dying, but these services are not universal and although studies have examined whether such services can prevent acute hospital admission, it is unclear whether they can prevent emergency hospice admissions (Buck, Webb, Moth et al., 2018; Ward, Graham \& Sixsmith, 2017).

Aim To investigate whether the introduction of a $\mathrm{H} @ \mathrm{H}$ service would reduce emergency hospice admissions, by analysing the needs of patients admitted as an emergency to a hospice inpatient unit.

Method All patients admitted as an emergency to this hospice, which does not currently offer a $\mathrm{H} @ \mathrm{H}$ service, are reviewed by the multi-disciplinary team at Mortality and Morbidity ( $\mathrm{M}$ and M) meetings. The data from this evaluation was collected between September 2016 and August 2017.

Results 190 emergency admissions took place during the above period. 93 patients (48.9\%) were admitted for complex symptom control, 52 patients $(27.4 \%)$ were admitted for end of life care, and 27 (14.2\%) were admitted for both complex symptom control and end of life care. Eleven patients $(5.8 \%)$ were admitted for multiple reasons including complex symptom control, end of life care, complex social situation and psychological distress. Seven patients (3.7\%) were admitted because of social crisis. At the $\mathrm{M}$ and $\mathrm{M}$ review it was agreed that a H@H service would not have prevented 122 admissions (64.2\%) but may have prevented 58 admissions (30.5\%). In 10 cases $(5.3 \%)$ it was unclear if $\mathrm{H} @ \mathrm{H}$ could have prevented admission.

Conclusion Most emergency admissions were for symptom control or multiple reasons, not end of life care alone; this may explain why only $30.5 \%$ of admissions could have been prevented with a $\mathrm{H} @ \mathrm{H}$ service. Patients choosing the hospice as their preferred place of death, or who were not imminently dying, will also affect the number of preventable admissions. This information on the needs of patients requiring emergency admission may help in the future development of a $\mathrm{H} @ \mathrm{H}$ service.

\section{P-155 INTRODUCING RAPID RESPONSE FOR COMMUNITY PALLIATIVE CARE, CLINICAL NURSE SPECIALISTS (CNS) TEAMS}

Geraldine Barry, Marika Killilea. North London Hospice, London, UK

10.1136/bmjspcare-2018-hospiceabs. 180

Background There is a national drive to support patients in their preferred place of choice and a need to reduce the number of unnecessary hospital admissions (Department of Health, 2008; National Palliative and End of Life Care Partnership, 2015). The community teams received over 1600 referrals last year. The triage team perform a telephone assessment, prioritising patients on need and transferring them to community clinical nurse specialists (CNSs). There was no formal pathway to respond to urgent needs of new and existing patients. It was unclear where to escalate urgent calls if all CNSs were occupied with planned visits. We decided to introduce a designated daily rapid response CNS into each of the teams to respond to the urgent referrals and reduce the number of patients who died before assessment.

Aims To introduce changes in working practices to respond to patients in a timely manner, with the intention to provide palliative care to more patients at home, thus supporting them in their preferred place of care (Calanzani, Higginson, Gomes, 2013).

Results The role of the rapid response CNS was defined and introduced into the three borough teams - each team identifies a rapid response CNS each day on the off duty. They are the first point of contact for the triage team and community administrators who frequently take calls from patients. The rapid response CNSs minimise planned visits for that day to ensure they have time to respond to urgent patient need.

Conclusion This was a challenge to traditional ways of working (Leary, Crouch, Lezard et al., 2008), however, involving the CNSs in developing the structure of the role and the pathway helped to develop a sense of ownership of the project. Although it is too early to formally evaluate there is a feeling of confidence and security for staff in the knowledge that their responsibilities are clearly defined. For the future we will examine responsiveness by introducing new activity codes to support data collection.

\section{P-156 URGENT RESPONSE: MEETING NEED, CHANGING CULTURE}

Nicola Griffiths, Fran Moffat, Sharon Hudson. Birmingham St Mary's Hospice, Birmingham, UK

10.1136/bmjspcare-2018-hospiceabs. 181

Background A Pilot Urgent Response Service was launched by the Hospice@ Home team due to it being identified that the nature of care needs for patients in the community were changing. More people required an urgent response for care crisis, symptom management, emotional distress and end of life care.

Aim The service aimed to support the Clinical Nurse Specialist and Primary Care Team in managing patients with urgent palliative care needs by coordinating a visit within two hours of a referral. The service was available to any patient already known to the Community Palliative Care Team (CNS, Hospice (a) Home and Day Hospice). 